\title{
Simultaneous \\ Microscopic Imaging of Elastic and Thermal Anisotropy
}

\section{5th International Conference on NDE in Relation to Structural Integrity for Nuclear and Pressurized Components}

\author{
D.H. Hurley \\ K.L. Telschow
}

May 2006

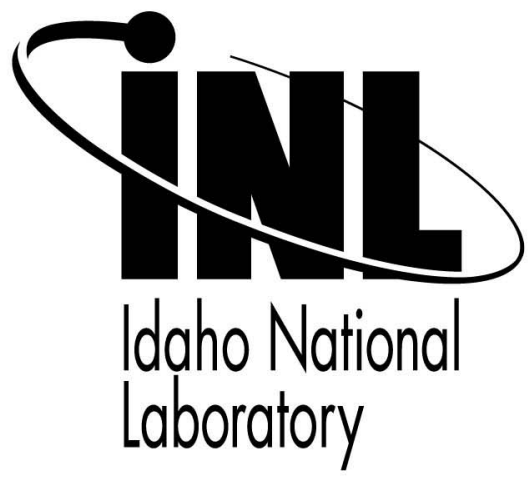

This is a preprint of a paper intended for publication in a journal or proceedings. Since changes may be made before publication, this preprint should not be cited or reproduced without permission of the author. This document was prepared as an account of work sponsored by an agency of the United States Government. Neither the United States Government nor any agency thereof, or any of their employees, makes any warranty, expressed or implied, or assumes any legal liability or responsibility for any third party's use, or the results of such use, of any information, apparatus, product or process disclosed in this report, or represents that its use by such third party would not infringe privately owned rights. The views expressed in this paper are not necessarily those of the United States Government or the sponsoring agency. 


\title{
Simultaneous microscopic imaging of elastic and thermal anisotropy
}

\author{
D. H. Huriey and K. L. Telschow \\ Idaho National Laboratory, P.O. Box 1625, Idaho Falls, Idaho 83415-2209, USA
}

\begin{abstract}
Simultaneous imaging of elastic and thermal properties of anisotropic materials with micron (lateral) and nanometer (depth) resolution is presented. This approach employs an ultrafast laser for the generation and detection of thermal and acoustic waves. Demonstrations involving the visualization of thermal waves and surface acoustic waves are presented for single crystal quartz and fused silica substrates sputter coated with chromium films. These images dramatically reveal and contrast the symmetry of thermal and elastic properties and compare favorably with theoretical prediction. This hybrid approach shows great promise to investigate fundamental properties of materials and interfaces on both a low-frequency (elastic wave) and a highfrequency (phonon diffusion) scale.
\end{abstract}

Accurate knowledge of thermal and elastic properties of materials at nanometer and micron length scales is germane to fully utilizing an increasingly large array of novel materials ranging from micromechanical devices to quantum well structures. For instance, a thorough understanding of thermal and elastic properties of thin metallic and dielectric films is required for effective thermal management and practical structural design in microelectronic and microphotonic devices. A great number of laser-based thermal waves studies $^{1-3}$ and laser-based acoustic studies ${ }^{4-6}$ have been conducted to image, separately, the thermal and mechanical properties of materials. Concerning point measurements, Capinski and Maris ${ }^{7}$ have employed an optical pump probe technique to study thermal conductivity in silicon and Hostetler et al. ${ }^{8}$ have simultaneously measured the elastic bulk modulus and the thermal diffusivity normal to the surface of thin films by combining picosecond acoustic and thermoreflectance techniques.

In this paper, a laser-based microscope for simultaneously imaging thermal and elastic anisotropy in nanometer-scale thin film-substrate systems is described. This hybrid photothermal-photoacoustic microscope utilizes a modelocked Ti:Sapphire laser to generate (pump) and image (probe) thermal and surface acoustic waves (SAW's) and has a depth resolution of a few tens of nanometers ${ }^{9}$ and a lateral resolution of a few microns. The laser system is operated at $800 \mathrm{~nm}$, has a repetition rate of $76 \mathrm{MHz}$, a pulse duration $\sim \mathrm{l}$ ps, and a pulse energy $\sim 0.1 \mathrm{~nJ}$. The pump and probe beams are focused, at normal incidence onto an optically absorbing film from opposite sides of a transparent substrate, through two microscope objectives producing spot sizes $D$ $\sim 3 \mu \mathrm{m}$ [Fig. 1(a)]. The probe leg can be set up either to measure changes in surface reflectivity, $\Delta R$, or to measure a combination of changes in surface reflectivity, $\Delta R$, and optical phase, $\Delta \varphi_{\mathrm{I}}$. The pump light is amplitude modulated using an AOM and lock-in detection is employed. The pump beam is scanned in the $x_{1}-x_{3}$ plane at a fixed pump-probe delay time, forming an image of lock-in amplitude $\left(A_{\mathrm{L}}\right)$ or lock-in phase $\left(\varphi_{L}\right)$. Table I gives a concise description of the various modes of operation that will be considered in this paper. Sorting out the wealth of thermal and elastic property infor- mation is accomplished by considering the amplitude dependence of each signal component as a function of modulation frequency. The SAW amplitude is related to the thermal gradient in the film imposed by optical absorption of the ultrafast optical pulse and is generally independent of the pump modulation frequency. ${ }^{10}$ In contrast, the amplitude of
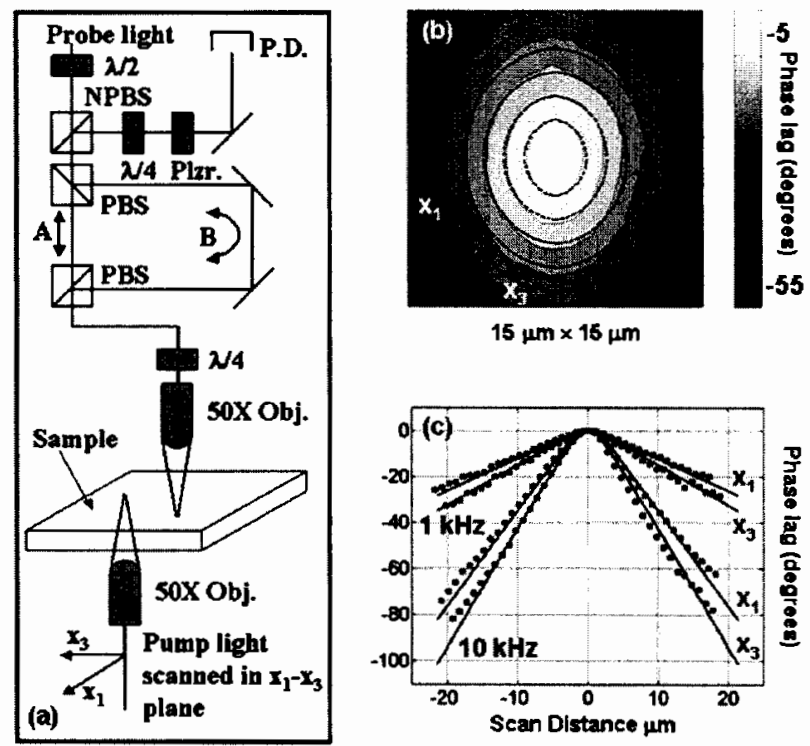

FIG. 1. (a) Experimental setup. The common path differential detection scheme is composed of two beams: one that traverses path $A$ and one that traverses path $B$. Phase demodulation is achieved using a $\lambda / 4$ wave plate and a polarizer placed before the photodetector. (b),(c) The lock-in phase image, $\varphi_{\mathrm{L}}$, and phase profile of the surface temperature variation were obtained for a 200-nm sputter-coated chromium film on a single crystal quartz substrate (the crystal $c$ axis coincides with the laboratory $x_{1}$ axis). The asymmetry in the phase profile and/or image relates to the anisotropic thermal conductivity tensor of the substrate. (b) The phase contour of the surface temperature at $3 \mathrm{kHz}$. The theoretical results are represented by the dashed lines. (c) The phase profiles at 1 and $10 \mathrm{kHz}$. The theoretical results are represented by solid lines. 
TABLE I. Tabular description of the low frequency $(1-10 \mathrm{kHz})$ and high frequency $(>100 \mathrm{kHz})$ operational modes. TW, AS, and AD refer to thermal wave, acoustic strain, and acoustic displacement, respectively.

\begin{tabular}{lll}
\hline \hline MODE OF OPERATION & \\
\hline & Lock-in amplitude $A_{L}$ & Lock-in phase $\varphi_{L}$ \\
\hline $\begin{array}{l}\text { Change in } \\
\text { reflectivity }\end{array}$ & $\Delta R(\mathrm{TW})^{\mathrm{a}}$ & $\Delta R(\mathrm{TW})^{\mathrm{a}}$ \\
& & {$[$ for LFO see } \\
& & Fig. 1(b)] \\
Interferometer & $\Delta R(\mathrm{TW})$ & $\Delta R(\mathrm{TW})$ \\
output & & \\
& $\varphi_{1}(\mathrm{AD})^{\mathrm{b}}$ & $\varphi_{\mathrm{I}}(\mathrm{AD})$ \\
& {$[$ for HFO see } & {$[$ for LFO see } \\
& Fig. 2(a) $]$ & Figs. 2(c) and 2(d)] \\
\hline \hline
\end{tabular}

${ }^{a}$ The change in reflectivity associated with photoelastic coupling, $\Delta R$ (AS), is determined from experiment to be more than an order of magnitude smaller than that associated with photothermal coupling, $\Delta R$ (TW), for high-frequency operation (HFO), and this disparity is amplified for low-frequency operation (LFO).

${ }^{b}$ For low frequency operation, the lock-in amplitude associated $\varphi_{I}(\mathrm{AD})$ is determined from experiment to be more than an order of magnitude smaller than the lock-in amplitude associated $\Delta R$ (TW). For high-frequency operation, these two signals are of comparable magnitude.

the thermal wave component diminishes with increasing modulation frequency. ${ }^{11}$ Moreover, for a film thickness $\sim 100 \mathrm{~nm}$, the film can be considered thermally thin for $\mathrm{kHz}$ range modulation (sampling both film and substrate properties) and thermally thick for $\mathrm{MHz}$-range modulation (predominantly sampling film thermal properties). Thus by judicially picking the modulation frequency and choosing the appropriate detection scheme, one can either primarily examine thermal waves or surface acoustic waves or simultaneously monitor both.

The first approach considered, Fig. 1, involves imaging the phase lag, $\varphi_{\mathrm{L}}$, of the temperature field due to periodic heating, in the $\mathrm{kHz}$ range, of the surface of a single crystal quartz substrate sputter coated with a $200-\mathrm{nm}$ chromium film. In this case the probe beam, ${ }^{12}$ which travels solely along path $A$ [Fig. 1(a)], senses small changes in surface reflectivity, $\Delta R$, caused by changes in temperature (modulated reflectance). An image of the phase lag, clearly revealing the presence of thermal anisotropy, is shown in Fig. I(b). The orientation of the single crystal quartz substrate is specified by a crystal plane perpendicular to the sample normal $(h, k, i, l)=(20,25,-9,1)$ and a crystal direction parallel to the laboratory $x_{1}$ axis $[u, v, t, w]=[-14,13,1,0] .^{13}$ For the purpose of visualization this orientation roughly corresponds to the crystal $c$ axis coinciding with the laboratory $x_{1}$ axis and the direction between the $c$ axis and the hexagonal edge coinciding with the surface normal (laboratory $-x_{2}$ axis.) In the laboratory reference frame the nonzero components of the conductivity tensor are, $\kappa_{11}, \kappa_{22}, \kappa_{33}, \kappa_{23}$. For this orientation the principle section of the representation quadratic is aligned with the laboratory $x_{1}$ and $x_{3}$ axes and hence, the phase image exhibits symmetry about the $x_{1}$ and $x_{3}$ axes. In order to compare the experimental results with theory consider the case of a thin, thermally isotropic, optically absorbing film on a thermally anisotropic, optically transparent substrate. Letting $\widetilde{T}=1 / 2 T \exp (i \omega t)+\mathrm{c} . \mathrm{c}$, , the heat diffusion equations for the film (subscript $f$ ) and substrate (subscript $s$ ) are given by

$$
\begin{gathered}
\kappa_{f} T_{f, i i}-i \omega \rho_{f} C_{f} T_{f}=Q_{0} e^{-x_{1}^{2} a_{1}^{2}-x_{3}^{2} a_{3}^{2}+\alpha\left(x_{2}-h\right)} \\
\kappa_{11} T_{s, 11}+\kappa_{22} T_{s, 22}+\kappa_{33} T_{s, 33}+2 \kappa_{23} T_{s, 23}-i \omega \rho_{s} C_{s} T_{s}=0
\end{gathered}
$$

where $T, \kappa, \omega, \rho, C, Q_{0}, a, h$, and $\alpha$ are the temperature, conductivity tensor, modulation frequency, density, specific heat, absorbed laser energy, laser spot size, film thickness, and the optical absorption coefficient, respectively. Note that in order to mimic the experimental setup the pump is introduced from the substrate side of the film. The thermal boundary conditions are given by

$$
\begin{gathered}
\kappa_{f} T_{f, 2}=\left.0\right|_{x_{2}=0}, \kappa_{f} T_{f, 2}=1 /\left.R_{\mathrm{th}}\left(T_{s}-T_{f}\right)\right|_{x_{2}=h}, \\
\kappa_{f} T_{f, 2}=\kappa_{22} T_{s, 2}+\left.\kappa_{23} T_{s, 3}\right|_{x_{2}=h},
\end{gathered}
$$

where $R_{\mathrm{th}}$ is the thermal contact resistance between the film and the substrate. The solution to these equations is sought by first applying a double Fourier transform to eliminate dependence on $x_{1}$ and $x_{3}$. The resulting ordinary differential equations in $x_{2}$ are solved analytically subjected to the boundary conditions, Eq. (2). The transformed solutions are then inverted numerically. The solution is represented by isothermal contour lines at $3 \mathrm{kHz}$ [Fig. 1(b)] and phase profiles at $\mathrm{l}$ and $10 \mathrm{kHz}$ [Fig. 1(c)]. The variation of a number of material parameters may be considered in order to obtain a fit between experiment and theory. ${ }^{14}$ Bulk thermal properties were used for the substrate, ${ }^{15}$ and considering the excellent adhesive properties typical of chromium films on silica substrates, the thermal contact resistance was set to zero. ${ }^{16}$ The film thickness, $200 \mathrm{~nm}$, was measured using a point-mode picosecond acoustic technique and the spot sizes of the pump and probe, $-3 \mu \mathrm{m}$, were measured using a knife-edge technique. Using bulk values for the film density and specific heat, a fit to within experimental error yielded a thermal conductivity of $40 \mathrm{~W} / \mathrm{mK}$, which is less then half the bulk value. This supposition is in keeping with previous studies that reported a decrease in film diffusivity with decreasing film thickness. ${ }^{17,18}$

The second approach, first presented by Sugawara et al., ${ }^{6}$ employs a common-path interferometric detection scheme ${ }^{19}$ for imaging changes in optical phase, $\Delta \varphi_{\mathrm{I}}$, associated with the propagation of acoustic waves along the sample surface. For this differential detection scheme, the optical phase is primarily sensitive to out-of-plane particle velocity. ${ }^{6,20}$ This mode of operation is employed to image surface acoustic waves using a modulation frequency of $1 \mathrm{MHz}$, Fig. 2(a) (sample specifications and orientation are the same as those 

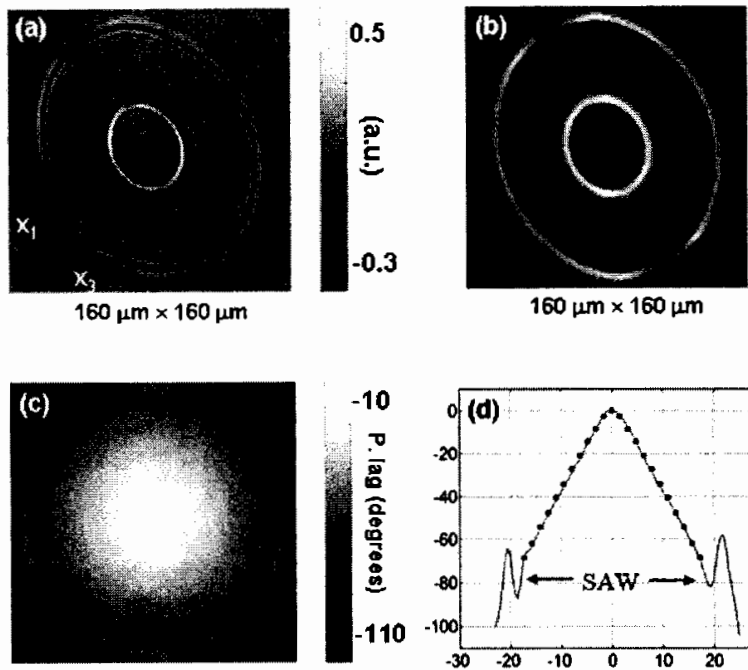

$23 \mu \mathrm{m} \times 23 \mu \mathrm{m}$
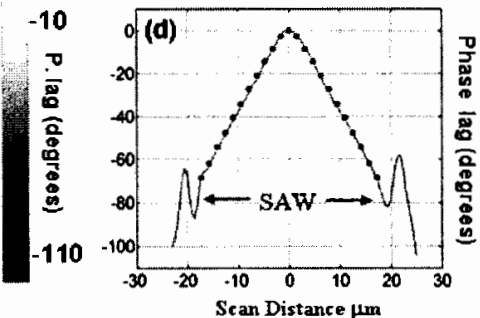

FIG. 2. (a) Interferometric image of variations in lock-in amplitude, $A_{\mathrm{L}}$, caused by surface acoustic and bulk waves propagating along the surface of a single crystal quartz substrate sputter-coated with a 200-nm chromium film (modulation frequency of $1 \mathrm{MHz}$ ). (b) Image derived from theory using an angular spectrum of plane waves. (c) The interferometric image of variations in the lock-in phase, $\varphi_{\mathrm{L}}$, caused by thermal and surface acoustic waves (fused silica substrate with a $200 \mathrm{~nm}$ chromium film.) (d) The cross section of the phase data presented in (c). The linear variation in the phase near the center of the image relates to the thermal wave. The rapid variation in the phase that forms a halo around the outside of the image is due to interferometric variations in the lock-in phase caused by the surface acoustic wave.

described in relation to Fig. 1). The concentric wave fronts, due to the 76- $\mathrm{MHz}$ periodic excitation of the laser source, reveal distinctive phonon focusing. ${ }^{21}$ The thermal wave signal at $1 \mathrm{MHz}$, which extends only a few microns and is dominated by the thermal properties of the film, is removed to improve the contrast of the surface acoustic wave image. To understand the contributions to this image from the various acoustic modes propagating along the surface of the sample, a relatively simple model of SAW's propagating on a trigonal material with arbitrary orientation was developed. The model accounts for acoustic diffraction from a point source by using an angular spectrum of plane waves. ${ }^{22}$ The secular equation for acoustic angular dispersion is given by

$$
\begin{gathered}
\left|\Gamma_{j k}-\delta_{j k} \rho v^{2}\right|=0, \\
\Gamma_{j k}=k_{i} k_{l} C_{i j k l}
\end{gathered}
$$

where $C_{i j k k}, \rho, v$, and $k_{i}$ are defined as the elastic stiffness matrix, the density, the acoustic phase velocity, and wave vector. A monochromatic SAW with planar wave fronts is assumed to have the form

$$
u_{i}\left(\theta_{n}\right)=\sum_{n=1}^{3} C_{n} \alpha_{i}^{n} e^{\left[i\left(k_{1}, x_{1}+k_{2}^{n} x_{2}-\omega t\right)\right]},
$$

where $C_{n}$ are weighting factors, and $\alpha_{i}$ are the eigenvectors that satisfy the boundary conditions

$$
T_{2 j}=C_{2 j k l} u_{k, l}=0 \quad \text { at } x_{2}=0 .
$$

In this formulation, the propagation vector is always along $x_{1}$ axis and the crystal is rotated about the surface normal to obtain an angular spectrum of plane waves that is used to approximate a point source

$$
v_{2}=\frac{1}{N} \sum_{n=1}^{N} v_{2}\left(\theta_{n}\right)
$$

Instead of a monochromatic SAW, the spatial profile of the out-of-plane particle velocity, $v_{2}$, was assumed to be a unipolar spike, ${ }^{23}$ which was convolved with a Gaussian to simulate pulse broadening due to a finite generation spot size. The two concentric surface acoustic waves (separated by 13.15 ns) and the bulk longitudinal wave compare closely with the experimental results, both in shape and in amplitude. Figures 2(a) and 2(b) reveal the rich character of the elastic tensor, namely that the SAW wave front, contrary to the thermal wave phase image, does not exhibit symmetry about the $x_{1}$ and $x_{3}$ axes. Note that the frequency-dependent dispersion, evident in the outermost SAW wave front, is not considered in the analysis. ${ }^{9}$

The third approach involves simultaneous imaging of surface acoustic and thermal waves. In this capacity, the interferometer measures optical phase changes associated with the propagation of acoustic waves, ${ }^{24} \Delta \varphi_{\mathrm{I}}$, and reflectivity changes associated with thermal waves, $\Delta R$. To emphasize the consequence of elastic and thermal anisotropy a thermally and elastically isotropic material system is chosen (fused silica substrate-200-nm chromium film.) In order to reveal the thermal character of the substrate, a $3-\mathrm{kHz}$ modulation frequency was selected. At this frequency the thermal wave signal dominates the surface acoustic wave signal; however, by monitoring the lock-in phase, $\varphi_{\mathrm{L}}$, instead of the lock-in amplitude, $A_{\mathrm{L}}$, both the thermal wave and the surface acoustic wave are imaged with comparable signal contrast. The information contained in Fig. 2(c) is best understood by considering a cross section of the phase image in Fig. 2(d). The linear variation in phase near the center of the image relates to the thermal wave component of the signal. The rapid variation in the phase signal at approximately $20 \mu \mathrm{m}$ from the center [halo around the outside of the image in Fig. 2(c)] is due to interferometric variations in a signal phase caused by the surface acoustic wave. The circular symmetry in the lock-in phase image, Fig. 2(c), is a direct result of thermal and elastic isotropy. It should be noted that to clearly separate thermal and elastic effects, the thermal and acoustic responses must be spatially distinct. In principle this is achieved by changing the optical path length of the pump relative to the probe so as to move the SAW further from the origin. However, the periodic laser excitation at 76 $\mathrm{MHz}$ places a limit on the extent to which one is able to 
spatially separate the thermal and elastic responses. A numerical simulation of the phase profile is represented by the open squares in Fig. 2(d). As with the chromium film-single crystal quartz sample, a fit to within experimental error was obtained using a film thermal conductivity of $40 \mathrm{~W} / \mathrm{m} \mathrm{K}$.

In summary, simultaneous imaging of elastic and thermal properties of thin film and/or substrate systems has been presented. This approach uses an ultrafast laser for generation and detection of thermal and acoustic waves and has two distinct modes of operation. The first imaging mode monitors the phase lag of the surface temperature by recording small temperature-induced changes in surface reflectivity. A second complimentary approach utilizes an interferometric detection scheme to image both the thermal wave phase profile and the out-of-plane particle velocity associated with the propagation of acoustic waves along the sample surface. Visualization of dynamic thermal and elastic properties at the nanometer scale will provide insight into fundamental mechanisms of phonon-related material properties. Future applications will allow detailed investigation of interface properties, such as Kapitza resistance, that are very difficult to make by other means.
'W. B. Jackson, N. M. Amer, C. Boccara, and D. Fournier, Appl. Opt. 20, 1333 (1981).

${ }^{2}$ J. Opsal, A. Rosencwaig, and D. L. Willenborg, Appl. Opt. 22, 3169 (1983).

${ }^{3}$ J. Hartmann, P. Voigt, and M. Reichling, J. Appl. Phys. 81, 2966 (1997).

${ }^{4}$ J. W. Wagner, Mater. Eval. 44, 1238 (1986).

${ }^{5}$ K. L. Telschow, V. A. Deason, R. S. Schley, and S. M. Watson, J. Acoust. Soc. Am. 106, 2578 (1999).

${ }^{6}$ Y. Sugawara, O. B. Wright, O. Matsuda, M. Takigahira, Y. Tanaka, S. Tamura, and V. E. Gusev, Phys. Rev. Lett. 88, 185504 (2002).

${ }^{7}$ W. W. Capinski, H. J. Maris, E. Bauser, I. Silier, M. Asen-Palmer, T. Ruf, M. Cardona, and E. Gmelin, Appl. Phys. Lett. 71, 2109 (1997).

${ }^{8}$ J. L. Hostetler, A. N. Smith, and P. M. Norris, Int. J. Thermophys. 19, 569 (1998).

${ }^{9}$ Films as thin as a few tens of nanometers can alter the character of the thermal wave and surface acoustic wave component of the signal.

${ }^{10}$ Large lateral thermal gradients require tight focusing of the pump beam $(<5 \mu \mathrm{m})$. Acoustic modes that reverberate in the film are only significant when the pump and probe are coincident.

"The amplitude of the thermal wave diminishes with modulation frequency according to $e^{-x / \bar{\omega} / 2 \kappa}$, where $\kappa$ is the thermal diffusivity.

${ }^{12}$ The term pump probe only strictly applies to the acoustic imaging mode of operation.

${ }^{13}$ The crystal orientation was obtained using electron backscatter microscopy.
${ }^{14} \mathrm{~B}$. Li, L. Pottier, J. P. Roger, and D. Fournier, Rev. Sci. Instrum 71, $2154(2000)$

${ }^{15}$ J. F. Nye, Physical Properties of Crystals (Oxford University Press, Oxford, 1985).

${ }^{16}$ It should be noted that had the thermal contact resistance been considered, a closer inspection of the governing equations would reveal that the anisotropic nature of the problem imposes a restraint on choosing the contact resistance. Namely, the theory should correctly predict the difference in the slope of the phase lag along the $x_{1}$ and $x_{3}$ directions. This restriction is understood by considering the limiting case of a large contact resistance. In that case, the film would carry the majority of the thermal energy, thus masking the anisotropic nature of the substrate.

${ }^{17}$ P. Nath and K. L. Chopra, Thin Solid Films 18, 29 (1973).

${ }^{18}$ G. Langer, J. Hartmann, and M. Reichling, Rev. Sci. Instrum. 68, 1510 (1997).

${ }^{19}$ D. H. Hurley and O. B. Wright, Opt. Lett. 24, 1305 (1999).

${ }^{20}$ From experiment, the photoelastic contribution to the signal, $\Delta R$ (acoustic strain) was determined to be an order of magnitude smaller than the optical phase contribution, $\varphi_{1}$ (acoustic displacement).

${ }^{21}$ G. W. Farnell and E. L. Adler, in Physical Acoustics, edited by W. P. Mason and R. N. Thurston (Academic, New York, 1972).

${ }^{22}$ M. S. Kharusi and G. W. Farnell, J. Acoust. Soc. Am., 48. 665 (1970).

${ }^{23}$ D. H. Hurley, J. B. Spicer, J. W. Wagner, and T. W. Murray, Uitrasonics 36, 355 (1998).

${ }^{24}$ The optical phase imposed on both signal beams (path $A$ and path $B$ ) by the temperature field is identical and hence, there is no thermal wave contribution to the optical phase. 